\title{
THE IMPORTANCE OF THE INITIAL POSTPARTUM/ POSTNATAL HOUR AND SKIN-TO-SKIN CONTACT - A TIME FOR CHANGE IN BULGARIA. \\ Ivanichka Serbezova ${ }^{1}$, Daniela Lyutakova ${ }^{2}$
}

\begin{abstract}
:
INTRODUCTION: This paper explores the practices surrounding skin-to-skin contact of mother and child immediately after birth within Bulgarian maternity wards and describes women`s experiences. It takes into consideration the research of Bulgarian experts in breastfeeding and contemporary recommendations for postnatal care worldwide.

Obstetric practices in local maternity wards are reviewed and evidenced by the research findings. The results put into perspective the different experiences that Bulgarian mothers have in local wards, and the authors explore these women`s attitudes towards the routine implementation of skin-to-skin contact in postnatal care.

The paper also outlines setbacks for introducing the practice in Bulgarian maternity wards due to the lack of midwife-led care and the importance of specialized care in optimizing health and a better understanding of skin-to-skin care.

OBJECTIVES: Purposes of this research are: (1) to explore women's knowledge about skin-to-skin benefits, including their past experiences with the practice, and (2) to assess their own personal motivation and willingness to engage in the practice themselves.

METHODS: The methodology applied includes an online-based anonymous survey that aims to explore parents' current levels of knowledge and gather their viewpoints regarding the practice. The research has been conducted on social media channels between the $26^{\text {th }}$ of January 2020 and the $26^{\text {th }}$ of February 2020 and includes women from varying local parent support groups: both respondents from a focus group, the area of Ruse, Bulgaria, and respondents from other major Bulgarian cities. The research findings are illustrated herein, and the authors discuss attitudes, as evidenced by the respondents' opinions, expressed through the survey.

RESULTS: A total of 771 cases are included, which come from both groups. The research demonstrates common practices in local hospital wards are inconsistent with WHO recommendations and evidence-based medicine. Skin-to-skin care is practically a non-existent practice within immediate postnatal care, with most mothers separated from their baby during this crucial first hour(s). Almost all the women surveyed are willing to engage in, and embrace, skin-to-skin practices to be introduced as part of the routine within local maternity wards.

CONCLUSION: Midwives' numbers in hospitals are dwindling, and women identify this is a worrying trend for the success of skin-to-skin bonding for new mothers. Evidence-based medical research and parental opinion compel us to rethink current postnatal practices, and therefore it is logical to suggest sustainable and realistic strategies for promoting and implementation of effective guidelines.
\end{abstract}

UDC Classification: 618, DOI: https://doi.org/10.12955/pmp.v1.104

Keywords: skin-to-skin, birth, midwife, newborn, postpartum, postnatal, healthcare

\section{Introduction and Literature review}

Up to date recommendations and guidelines for immediate postpartum/postnatal care (hereinafter 'PP care') include practicing skin-to-skin contact as per WHO (2018), Flacking et al. (2012), Moore et al. (2016). It is advised skin-to-skin contact shared by a mother and her newborn (or the father if the mother is not active or responsive) should begin immediately after birth and not be interrupted. Also, that any necessary interventions that must be performed, taking into consideration the paramount importance of skin-to-skin care, be postponed or carried out while the mother and the infant are together as evidenced by Serbezova (2016), WHO (2018), Moore et al. (2016). Optimal care for mother and baby is the care that allows them to stay together, in skin-to-skin contact, until the first breastfeeding has finished as Moore et al. (2016) state. Observations and research by Moore et al. (2016), WHO (1998), Serbezova (2016), Popivanova (2016), show this practice has a positive role in early breastfeeding and has physiological benefits for the newborns' transition into the outside world, as evidenced by Moore et al. (2016). Additionally, it positively affects the infants' thermoregulation, minimizes pain and stress for the newborn (Serbezova (2016), Popivanova (2016), and has a significant positive effect on the neurological, psychological, and emotional development of the baby according to Flacking et al. (2012), Popivanova (2016). It also lowers the risk for psychological and or emotional issues for the mother after birth as stated by Popivanova (2016), Serbezova (2016). Science-backed medical research unequivocally supports the practice of immediate or early skin-to-skin contact (when a newborn infant is placed naked on the mother's bare chest). Experts are advised to encourage this method of care for healthy and full-term babies, disregarding whether they were born naturally or via a caesarean section,

\footnotetext{
${ }^{1}$ Ruse University „Angel Kanchev“, Faculty of Public Health and Health Care, iserbezova@uni-ruse.bg

${ }^{2}$ Ruse University „Angel Kanchev“, Faculty of Public Health and Health Care, lachezarova@gmail.com
} 
as stated by Popivanova (2016). Kangaroo care also includes skin-to-skin contact (SSC) within its principles and is performed for premature newborns as Serbezova (2016) highlights.

Clinical experience points to the worrying fact that in Bulgarian maternity wards, immediate or early SSC is practically non-existent. This leads us to conclude that further research in this matter is warranted. Observations that we share coincide with the results of a survey among over 5000 women that was conducted in 2016 by various associations and non-governmental organizations (NGOs). The survey found that a mere c. $14 \%$ of babies born in Bulgarian maternity wards are not separated from their mothers immediately after birth, and more than one-third of babies were breastfed for the first time after at least 6 hours had elapsed since birth, as per Sdruzhenie Rodilnitsa (2016).

In $86 \%$ of cases reported, SSC within the first-hour post-birth did not happen at all, and in half of those cases, the reason for this was the facility's own regulations, as stated by Sdruzhenie Rodilnitsa (2016). This motivates us to improve the situation for mothers and babies' alike and establish a sustainable strategy for implementing evidence-based practices, including SSC algorithms.

This research aims to analyze local women's overall knowledge and attitudes towards SSC and their personal experience with SSC. Thus, this enabling us to draw logical conclusions and developing a sustainable strategy to popularize and introduce the practice in local maternity wards. This would begin by addressing women's specific needs and views to assist in building effective algorithms.

\section{Data and methodology}

The study was conducted within the period of the $26^{\text {th }}$ of January 2020 through the $26^{\text {th }}$ of February 2020, utilizing a cross-sectional study design and collecting data through a self-administered online questionnaire, which includes both closed-ended and open-ended questions. This was directed predominantly at mothers participating in online communities with a focus on parenting.

At the first stage of the research, we have explored different local social-media groups, searching with the keywords, "mothers", "mums", "pregnancy", "birth", "breastfeeding" via the Facebook search engine. The target population for our study was members of national online support groups for current parents, specifically mothers. The questionnaire was based on a self-selected sample and was computerassisted. The online questionnaire was published as an accessible link in three closed Facebook groups with the approval of an administrator. The participation method for the study was random, and participants were reached exclusively via the Facebook platform.

The online survey has been specifically designed for the present research and consists of 2 sections and is a 12-point questionnaire, developed via Google Forms with a combination of a checkbox, multiplechoice, and free-text types of questions. They were presented to all participants in the exact same order, and providing an answer to a minimum of 10 of the questions was required.

The first section consisted of questions in regard to the demographic data of respondents, with the second one examining respondents' awareness about the practices and experiences with SSC. Respondents have filled out the survey voluntarily and anonymously online, filling out a different report for each childbirth. A total of 561 respondents were reported in the common group and a total of 210 in the focus group accumulating to 771 participants as being the combined number of all respondents in this research. The response rate for the common group is $1.94 \%$ and $1.6 \%$ for the focus group.

In the interpretation, we used the quantitative as well as qualitative analysis methods. Both authors reviewed the text responses and organized data from closed-end questions in Microsoft Excel Version 2016.

\section{Survey Results}

\section{First Segment - Demographics}

The first segment of the online survey aims to establish basic demographic data about the respondents. This helps us identify their profile and get a better understanding of the information we analyze. As expected, almost all women are in their reproductive age, which is unsurprising considering the theme of the groups we have surveyed. The biggest group of respondents is women aged 22-30 years (46.9\%), closely followed by 31-35 years (36.2\%). We have also included information about the respondents' parity which would give us more insight on their experience with SSC implementation. Data shows most respondents have at least one child (primiparous or multiparous) - a total of $96.1 \%$. As a part of the demographical segment of the research we also included a question regarding the women's education. 
Results show the group represents a well-educated population - a total of $77 \%$ of women who filled out the survey have a Bachelor's / Master's degree. Further details for the demographic data collected and respondents` characteristics are presented in Table 1.

\begin{tabular}{|c|c|c|c|c|c|c|c|c|}
\hline & Category 1 & $\%$ & Category 2 & $\%$ & Category 3 & $\%$ & Category 4 & $\%$ \\
\hline Age profile & $18-21$ & 2.1 & $22-30$ & 46.9 & $31-35$ & 36.2 & $\begin{array}{l}36-45 \text { or } \\
\text { over } 46\end{array}$ & 14.8 \\
\hline Parity & primiparous & 63.3 & multiparous & 32.8 & nulliparous & 3.9 & N/A & N/A \\
\hline $\begin{array}{l}\text { Educational } \\
\text { status }\end{array}$ & $\begin{array}{l}\text { Primary } \\
\text { and/or high- } \\
\text { school }\end{array}$ & 18.7 & $\begin{array}{l}\text { College } \\
\text { degree }\end{array}$ & 4.3 & BSc degree & 31.4 & MSc degree & 45.6 \\
\hline
\end{tabular}

\section{Second Segment - Awareness, Experience, Motivation}

The second segment of the research looks at women's knowledge and personal experiences regarding skin-to-skin care. The authors have included questions investigating both respondents' viewpoints on it and how they perceive their own experiences with the practice, if indeed they had the access to such care in the first place.

The first question of this section aims to establish how familiar women are with SCC. We have asked them to evaluate their knowledge as per their own views and or experience.

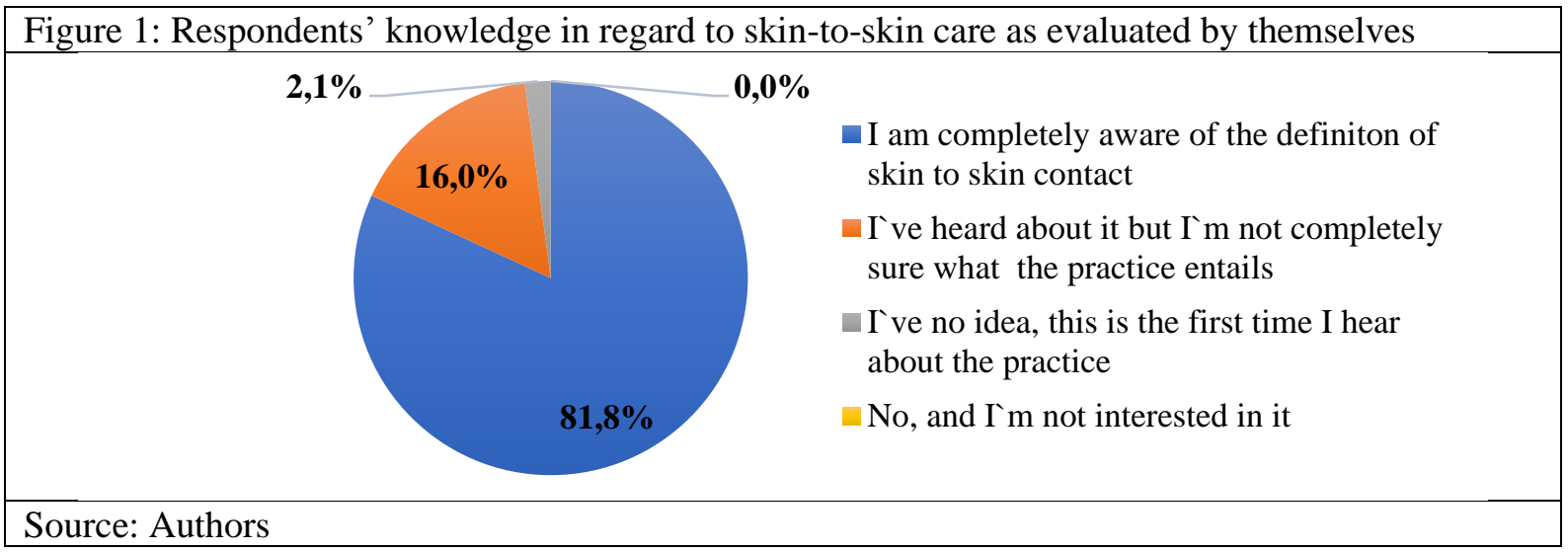

As evidenced by this data, most women state they are perfectly aware of what skin-to-skin care includes and none of the respondents shows any disinterest in it. This leads us to believe all respondents from the group view it as an essential aspect of maternity / PP care, and that new mothers need to be thoroughly informed about it. Only $2.1 \%$ heard about it for the first time and this supports the view that a sustainable and effective model of antenatal midwife-led care be found. The data shows $16.0 \%$ of women are not confident that they are familiar with this practice, which is a factor we have to consider when further interpreting the results from the survey.

This research also looks at the reasoning behind some women's views of the practice, looking at the motivation that leads them to believe SSC is such a necessary practice. We have given them several options to choose from (multiple choice questions) as to better understand how they prioritize the benefits of SSC immediately after birth.

The following data has been gathered, graphically presented in Figure 2, and tendencies are discussed below.

The women's answers evidence that they perceive the bonding process of SSC as the most significant benefit. It is ranked first with $86.8 \%$ selecting it. It is closely followed by $82.2 \%$ stating that skin-toskin offers an easier adaptation for their newborn. Benefits for the processes of breastfeeding and psychological well-being of the mother are not as popular, both gaining around c.50-60\% preference. The latter shows that many respondents feel they are perfectly aware what skin-to-skin care entails, but they still do not have the full facts of it. It is essential antenatal care that allows for enough preparation so that families have all the information on each practice within maternity care. In turn, they can make an informed decision. Only partially recognizing these SSC benefits deprives families of beneficial aspects within childbirth. 


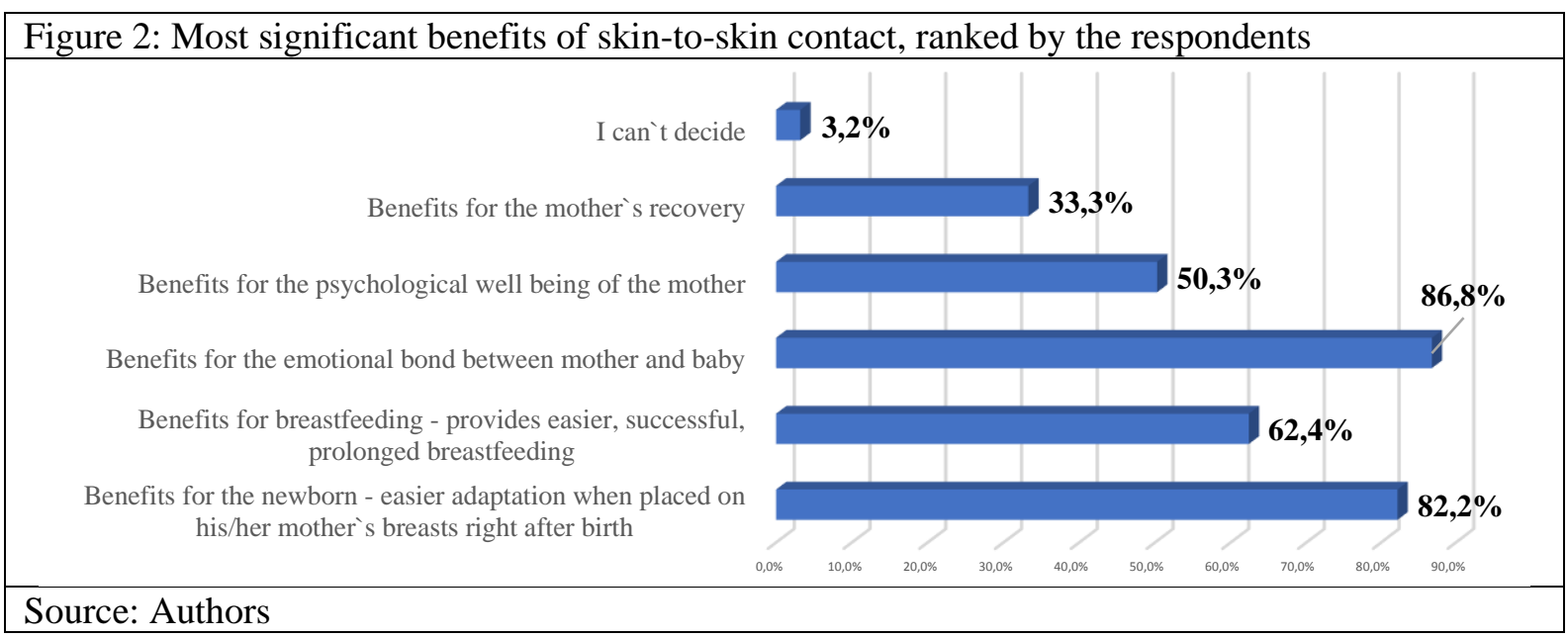

The next question we posed to women aimed at identifying weak-points in information campaigns and the respondents' fears and or uncertainties regarding to skin-to-skin care. This helped us formulate a more effective strategy for implementing the practice, allowing for optimal implementation of women's needs. Knowing what obstacles women observe give us insight on the way they perceive skin-to-skin care and whether they grasp the fundamental power it holds for a newborn. Breakdown in communication and lack of midwifery care models have deprived local women of a holistic view of maternity care and practices. We should be looking at the answers provided, consider the information they have access to and how they have found a way to access it. Quality of care includes antenatal preparation and classes for the families, but this has mainly been in the private sector. Namely, antenatal consultations have exclusively been ultrasound checks with obstetricians and any midwifery care is almost non-existent today.

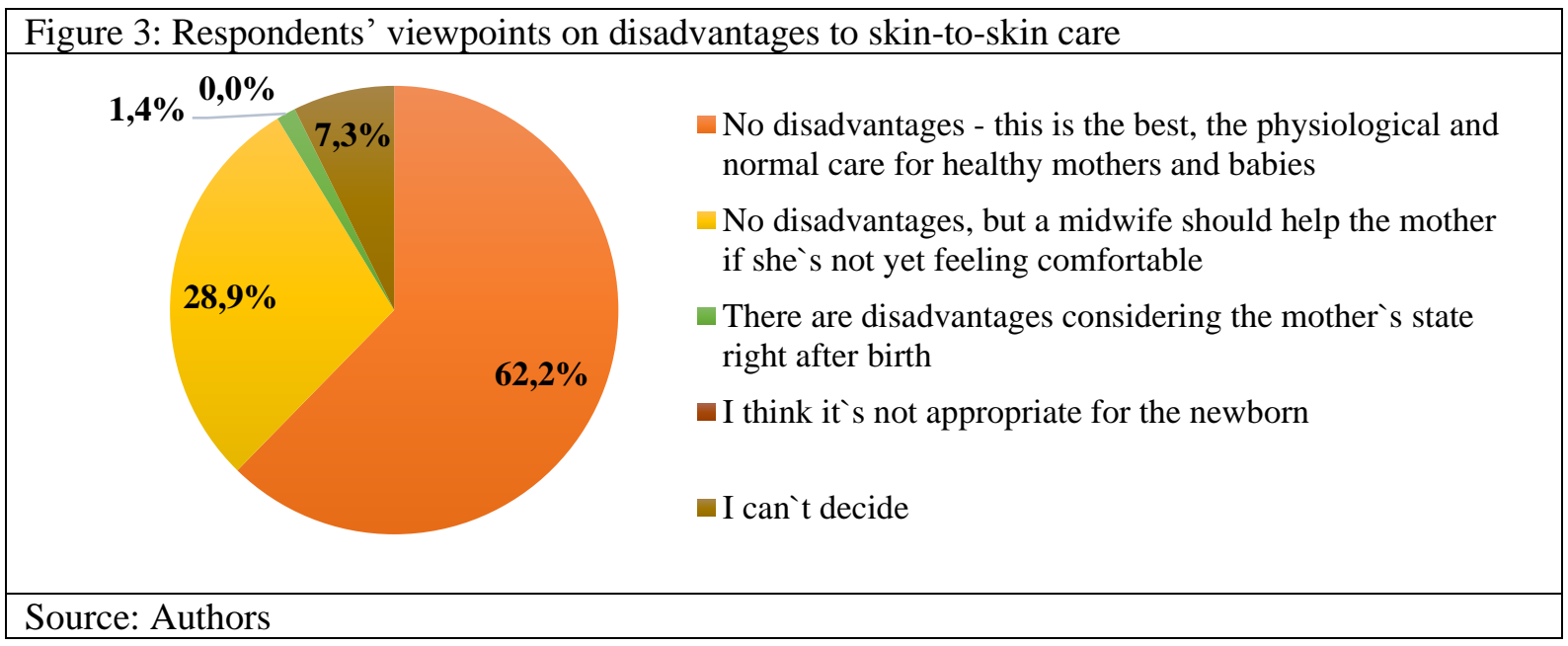

Data shows the most significant worry women face is regarding their state in the immediate PP care period, especially those who doubt their own capabilities during this period. The data shows $28.9 \%$ identify the need for a skilled midwife who can help guide them during this time and help with SSC. This supports the necessary review and or reorganization of care.

The global healthcare crisis and the deficit of midwifery nurses are having a direct effect on local maternity wards and the health of mothers and their children. Another factor worth considering is that women are routinely not allowed a birthing partner. In most maternity wards, there is even a fee for a birthing partner to attend, even fathers. Doulas are not allowed in most wards in the country, with a few exceptions in Sofia's wards. This leaves women feeling more vulnerable and leads to their own selfdoubts, insecurities, and lack of confidence in caring for their newborn. Midwifery-led care could easily solve this problem.

We also gave women the option to share some more thoughts about disadvantages in their own words. Some of the comments include: 
"Fathers need to be welcomed into labor wards, and after the birth, while mom and baby are still in the hospital. This makes the bond between them even stronger."

"Uneasiness because of all the strangers in the room."

"I think midwives should be next to the mom at all times, but lack of personnel interferes with their work."

"I was not prepared and when the time came, I did not ask them to have my baby immediately. When she was born, they immediately whisked her away for measurements. Like something bad will happen to baby if they postpone this. I am still angry and frustrated I did not have SSC with the baby."

"The practice itself doesn't have any disadvantages. The discomfort comes from having to fight and argue with the medical experts to have this contact with your own baby.

"The medical team doesn't want to do it. They could easily place the baby on the mother's skin. It is just that they are not acquainted with this practice and don't see it necessary. Pediatricians do not allow such care."

Each citation is from an individual respondent who expresses their thought(s) regarding the SSC practice.

One of the main goals of this research is to determine whether women are approving of SSC to be routinely introduced into local wards. As with any aspect of maternity care, women's needs must be acknowledged, and PP care experts must work on a sustainable strategy that allows the best standard of care immediately after delivery. Evidence-based medicine is essential to health and safety outcomes in health care. This needs to be thoroughly implemented, and internal guidelines should be updated in accordance with the same.

\begin{tabular}{|l|l|}
\hline Figure 4: Respondents' viewpoints on implementing skin-to-skin care in local wards \\
\hline $\mathbf{0 , 4 \%}$ \\
$\mathbf{1 9 , 1 \%}$ & $\begin{array}{l}\mathbf{1 , 4 \%} \% \text { Yes, this would be the best decision } \\
\mathbf{7 9 , 1 \%}\end{array}$ \\
$\begin{array}{l}\text { Yes, but I would like to decide depending on } \\
\text { my condition and to be supported by my } \\
\text { midwife } \\
\text { No, I don`t think it is necessary }\end{array}$ \\
\hline Source: Authors
\end{tabular}

Numbers show that nearly all women would like skin-to-skin care to be routinely implemented in local maternity wards, i.e. $98.2 \%$ of all respondents. This motivates us to actively work towards finding an effective strategy for implementing SSC in local maternity wards. It is our duty as birthing and scientific experts to ensure the best quality of care, especially when both evidence and women point us unambiguously in the right direction.

To better grasp the current situation in local maternity wards, we have asked women to share their own experiences. The following data has been gathered and is graphically presented in Figure 5.

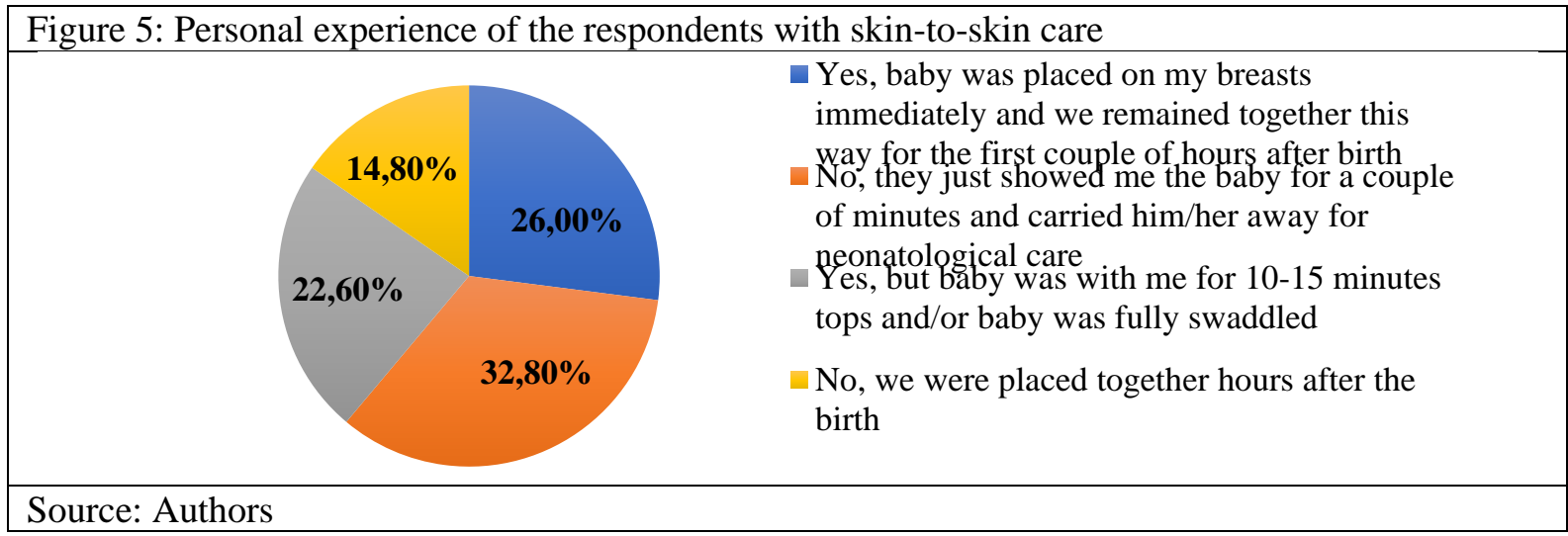


Statistics show women do not have routine access to skin-to-skin care in local maternity wards with around $67.2 \%$ of births not including the practice. Data from the survey shows women only see their newborn briefly before they are whisked away by the medical team for routine check-ups and measuring. Mothers stay for a minimum of 2 hours in a separate room so that they can be closely monitored, and babies are taken care by a separate medical team elsewhere.

\section{Focus Group Results}

The focus group comprises of 210 respondent cases who are currently participating in a closed Facebook group for peer support around Ruse, Northern Bulgaria. They have filled out the survey voluntarily and anonymously, filling out a different report for each childbirth. This focus group survey aims to identify whether local women share the same profile and viewpoints, and to compare data to the data from the common group.

Results are close to those of the common group and there are no substantial differences in women's viewpoints. Their demographic data are consistent with those of the common group: the share of cases in reproductive age is $76.2 \%$, with $21 \%$ in their late reproductive age. Data also points out most respondents have at least one child (primiparous or multiparous), i.e. a total of 93.4\%. The focus group also represents a well-educated population, i.e. a total of $65.7 \%$ of women reported have a bachelor's / master's degree. In regard to women's awareness about SSC, we can conclude that while in the common group $81.8 \%$ of women have knowledge on the matter, a decrease by almost $10 \%$ is demonstrated in the focus group $(72.9 \%)$, which is worth noting.

As for ranking SSC benefits, data from the focus group is also relevant to data in the common group. Benefits for the bonding process is again ranked first with $80.5 \%$ selecting, closely followed by $74.3 \%$ stating that SSC offers an easier adaptation to early life for their newborn. Benefits of the processes of breastfeeding and the psychological well-being of the mother are again not as popular amidst women, with both gaining around $41 \%$ preference.

Concerning SSC disadvantages, as stated by the respondents, we observe the same tendencies. Data shows $52.1 \%$ state that SSC has no disadvantages as compared to $62.2 \%$ in the common group. Data also evidence that the most significant uncertainty women are faced with is regarding their state in immediately PP care and self-doubt their capabilities during this period. $32.9 \%$ (as compared to 28.9\% in the common group) identify the need of a midwife.

We attach their thoughts concerning the disadvantages of SSC in their own words:

"If the mother is dizzy form an anesthesia or exhausted by a prolonged birth, this could be even more of a burden to her state."

"Lack of staff"

"Cannot be performed after a C-section"

"Mom is tired from birth. Baby never starts suckling this early."

"If a midwife can help me clean up after the birth and is with me in those first hours, I would definitely like to have skin-to-skin contact with the baby."

Data shows women would like skin-to-skin care to be routinely implemented in local maternity wards with a close percentile comparison, $98.6 \%$ vs. $98.2 \%$.

Data from the focus group points women do not routinely have access to SSC in local maternity wards with a total of $88.6 \%$ of births, not including the practice. In $16.7 \%$ of cases, mother and baby were placed together hours after the birth, and $49.5 \%$ (the biggest share of cases) reportedly were separated due to facility-based guidelines for immediate newborn care, e.g., immediate physical exams, measuring, swaddling, as compared to $22.6 \%$ share in the common group data.

\section{Discussion}

Women`s experiences are crucial to understanding the limitations of the current practices in Bulgarian maternity care. The objective of this study was to explore whether Bulgarian women are informed about SSC and its benefits, and whether they had any personal experience with such care and the level of their motivation to have access to this type of care in their future or impending births.

In our research, SSC was predominantly described by the respondents as a necessary and beneficial practice within the complex of immediate newborn care practices. Data obtained through our survey 
shows respondents have a satisfactory level of awareness on the topic and are actively interested in getting more information regarding the details and benefits of practicing SSC. Unfortunately, data also points to a low percentage of actual access to SSC in local hospitals. This is in line with findings from a previous study with a wider range conducted in 2016 by an NGO in the field of maternity and childcare, Sdruzhenie Rodilnitsa (2016).

Results from our study also demonstrate women recognize the practice as an essential part of maternity care and do identify midwives', namely a significant finding for the successful implementation of this type of care in local maternity wards. Mothers share their concerns that although they would like SSC included in their birth plans, it may not be adequate in every case since they are not confident in their capacity to safely establish skin-to-skin contact if a midwife is not present and available at all times during the earliest PP care. The shortage of staff is a key factor for current practices and directly influence how maternity care is organized. This is consistent with our observations regarding the dwindling numbers of midwives nationally and internationally, leading to an ineffective midwife to patient ratio.

As per the information published by the EU Commission in the Individual Country Health Profiles for 2019, Bulgaria has the second-lowest density of nurses in the EU, OECD, (2019). Stated in the same report, the average age of nurses and midwives is 55 years and there`s a marked tendency for migration amongst younger healthcare experts, additionally straining the system and multiplying the workload for those that stay and work within Bulgarian wards, OECD, (2019).

The lack of a clear birthing-companions' policy within Bulgarian wards further affects mothers` selfconfidence and positive experience intrapartum/ postpartum and is also a factor regarding women`s views on immediate newborn care practices / SSC. A companion of choice is recommended for all women throughout labor and childbirth, as per WHO (2018), and evidence shows this approach leads to a positive experience, empowering women during their birth, respectively, giving them better confidence in regard to SSC.

\section{Conclusion}

Developing adequate guidelines and algorithms for optimal newborn care is of paramount importance. This research showcases women's willingness to take part in this change and make informed decisions for their children's health. Birthing experts need to work on bridging the gap between evidence-based medicine, official recommendations, mothers` necessities, and the actual practices currently employed in Bulgarian maternity wards. Undoubtedly, midwives are in a strategic position to update expectant families' prenatal education and introduce information about SSC in the context of midwife-led care models. Thus, a sustainable pattern for the introduction and popularization of SSC can be achieved, implementing all the well-documented benefits by both scientific research and clinical experience alike.

\section{References}

Flacking, R., Lehtonen, L., Thomson, G., et al. (2012). Closeness and separation in neonatal intensive care.

Moore E.R., Bergman N., Anderson G.C., Medley N., (2016). Early skin-to-skin contact for mothers and their healthy newborn infants. Cochrane. Retrieved February 2020 from: http://www.cochrane.org/CD003519/PREG_early-skin-skincontact-mothers-and-their-healthy-newborn-infants

OECD/European Observatory on Health Systems and Policies (2019), Bulgaria: Country Health Profile 2019, State of Health in the EU, OECD Publishing, Paris/European Observatory on Health, Systems and Policies, Brussels. ISBN 9789264383685 (PDF), Series: State of Health in the EU, SSN 25227041 (online)

Popivanova, A., (2016) Kontakt ,kozha do kozha “ ili za neprekasnatiya kontakt mezhdy maykata i bebeto sled razhdaneto. $N A P K$ [The Skin-to-skin contact or for the undisturbed contact between mother and child after birth.] Retrieved Februray 2020, from: http://www.podkrepazakarmene.com/early-contact-878.html

Serbezova, I. (2016). Metodut „kozha do kozha“- metod za kachestvena sestrinska i akusherska grizha. Izvestiya na sayuza na uchenite $v$ Ruse, tom 6 . [The „Skin-to-skin“ method - a method for quality nursing and midwife care. Proceedings from The Union of the Scientists in Ruse, 6th Edition.]

Sdruzhenie Rodilnitsa (2016). Anketa za ustanovyavane na praktikite za PURVI KONTAKT - kratuk analiz na rezultatite. [The Skin-to-skin contact or for the undisturbed contact between mother and child after birth.] Retrieved February 2020, from: http://www.rodilnitza.com/blog/news-events/anketa-praktiki-1-kontakt/

WHO, (2018), WHO recommendations: intrapartum care for a positive childbirth experience. ISBN 978-92-4-155021-5. Retrieved February 2020, from: http://apps.who.int/iris/bitstream/handle/10665/43633/9241591544_eng.pdf?sequence=1 